\title{
Survival Outcomes and Factors Associated with Revision Surgery for Metastatic Disease of the Spine
}

\author{
Vignesh K. Alamanda $\mathbb{D}^{1},{ }^{1}$ Myra M. Robinson, ${ }^{2}$ Jeffrey S. Kneisl, ${ }^{3}$ \\ Leo R. Spector, ${ }^{4}$ and Joshua C. Patt ${ }^{3}$ \\ ${ }^{1}$ Department of Orthopaedic Surgery, Carolinas Medical Center, Atrium Health, Charlotte, NC, USA \\ ${ }^{2}$ Department of Cancer Biostatistics, Levine Cancer Institute, Atrium Health, Charlotte, NC, USA \\ ${ }^{3}$ Department of Orthopaedic Surgery, Levine Cancer Institute, Atrium Health, Charlotte, NC, USA \\ ${ }^{4}$ Ortho Carolina's Spine Center, Charlotte, NC, USA
}

Correspondence should be addressed to Vignesh K. Alamanda; vignesh.alamanda@gmail.com

Received 18 December 2017; Accepted 29 May 2018; Published 25 June 2018

Academic Editor: Akira Hara

Copyright (c) 2018 Vignesh K. Alamanda et al. This is an open access article distributed under the Creative Commons Attribution License, which permits unrestricted use, distribution, and reproduction in any medium, provided the original work is properly cited.

\begin{abstract}
Study Design. Retrospective review of a prospective database. Objective. Certain subset of patients undergoing surgical treatment for spinal metastasis will require a revision surgery in their disease course; however, factors predictive of revision surgery and survival outcomes are largely unknown. The goal of this study is to report on survival outcomes as well as factors predictive of revision surgery in this unique patient population. Methods. A total of 55 patients who met the inclusion criteria were included from January 2010 to December 2015. Twelve (22\%) of these patients underwent a revision surgery. Patient and tumor characteristics were summarized and survival outcomes were evaluated using Kaplan-Meier methods and Cox proportional hazards regression. Results. Both the revision and the nonrevision groups were similarly matched with respect to spine disease burden, neurological status at time of initial presentation, primary malignancy types, and the use of adjuvant treatment modalities. Tumor progression $(66.7 \%)$ was the most common reason for necessitating a revision followed by nonunion (16.7\%), wound dehiscence (8.3\%), and construct failure (8.3\%). Following multivariate model selection procedures, smokers were found to have 3.5 times increased odds of undergoing revision compared to nonsmokers $(p=0.05)$. Analysis of survival curves showed that the median survival in the revision group was 3.0 years (95\% CI: 1.5, 4.1), while the median survival in the nonrevision group was 1.5 years (95\% CI: 1.1, 2.3; log-rank test, $p=0.105)$. Conclusion. Despite aggressive treatment, tumor progression is the most common reason for revision surgery. Smoking is an independent risk factor for revision. Revision surgery should be considered in patients when indicated as it does not appear to detrimentally affect survival.
\end{abstract}

\section{Introduction}

The incidence of spinal metastasis continues to increase [1]: more than 18,000 new cases are reported every year in North America alone [2]. Treatment strategies and modalities continue to improve and have resulted in tremendous improvement in overall survival rates for these patients [3-5]. As overall survival rates continue to increase, the rate of skeletal and in particular spinal metastases can be expected to increase [6]. Additionally, indications for surgical intervention are also continuing to expand [7].
Tools for estimating prognosis have been developed to aid treating physicians with management options including surgical intervention $[8,9]$. When indicated, surgical treatment is not without its drawbacks. The need for revision surgery exists in this vulnerable patient population and reasons cited include surgical site infections and wound dehiscence, failure of instrumentation, and local recurrence [9]. However, not much has been established regarding the survival outcomes of those who undergo revision surgery and the factors that predispose certain patients to undergo revision surgery. 
Thus, in this study, we primarily sought to explore and compare the survival outcomes of patients undergoing revision surgery as compared to patients who only underwent a primary procedure. Additionally, our secondary goal was to assess and identify reasons for revision and risk factors that could predispose such patients to needing a revision.

\section{Methods}

After obtaining Institutional Review Board approval, a retrospective cohort study of a prospective database at a major cancer center was conducted to compare patient, tumor, and survival characteristics between those patients requiring a revision surgery and those who only underwent a primary surgical procedure.

Patients undergoing surgical treatment at our institution by the senior author between January 2010 and December 2015 were identified based on a retrospective review of a prospective database and were considered for the study. Exclusion criteria were age below 18 years and nonsurgical treatment for their metastatic disease to their spine. Additionally, primary tumors of the spine undergoing surgical treatment were excluded.

A total of 85 patients with a minimum of 6-month followup were identified and, after applying the exclusion criteria, 55 met the inclusion criteria. Patient demographics and tumor characteristics collected included age, gender, race, smoking status, primary malignancy type, number of vertebral levels affected, and location of spinal metastases. Additionally, treatment information, including use of radiation therapy and/or chemotherapy, use of preoperative embolization, and use of allograft, was abstracted from the medical record. The presence or absence of pain, the American Spinal Injury Association (ASIA) score at time of presentation, and reason for revision were also obtained. Overall survival (OS) was measured from the date of diagnosis of spinal metastasis to date of death from any cause. Surviving patients were censored at the date of last follow-up.

Frequencies and proportions of patient and tumor characteristics were reported and compared across groups using $t$-tests or Wilcoxon rank-sum tests for continuous variables and Fisher's exact tests for categorical data. Kaplan-Meier techniques were used to estimate OS distributions and a log-rank test compared the survival distributions between those with and without subsequent revision surgery. Cox proportional hazards regression was used to determine a hazard ratio (HR) and 95\% confidence interval (CI) to estimate the magnitude of the impact of revision surgery on OS. Univariate and multivariate logistic regressions assessed the impact of patient and disease characteristics on need for revision surgery and were used to determine odds ratios (OR) to estimate the magnitude of the impact of those factors on need for revision surgery. Multivariate logistic models were determined for the outcome of revision surgery (yes/no) using backward elimination and forward selection modeling procedures (significance levels of $p=0.10$ ). Individual prognostic factors were identified through univariate logistic models for all potential covariates (age, race, gender, smoking status, primary malignancy type, preoperative ambulatory status, radiotherapy, chemotherapy, and presence of extraspinal metastases).

Statistical software SAS (version 9.4) was used for all data analyses. Unless otherwise noted, a 2-sided $\alpha=0.05$ significance level was used.

\section{Results}

3.1. Table 1: Patient and Tumor Characteristics. Of the total of 85 patients that were operated on by the senior surgeon in the group from January 2010 to December 2015, 55 patients met inclusion criteria. Of these 55 patients, 12 patients $(21.8 \%)$ had undergone subsequent revision surgery.

Gender and race were not statistically different between the two groups. Approximately $60 \%$ of the study population was male and approximately $72 \%$ was Caucasian. In analyzing smoking between the two groups, $75 \%$ of the patients in the revision group were smokers compared to only $42 \%$ in the nonrevision group $(p=0.055)$. Renal cell carcinoma was the prominent cancer subtype in both groups $(41.7 \%$ and $23.3 \%$ in the revision and no revision groups, resp.).

Both groups had similar proportions of metastasis to the various spinal segments. In analyzing pain, no significant difference exists between the two groups: $100 \%$ of the revision surgery group had pain at time of initial presentation following diagnosis of spinal metastasis compared to $91 \%$ in the nonrevision surgery group. None of the patients who underwent a revision surgery had spinal metastasis present at time of initial cancer diagnosis compared to $28 \%$ of the patients in the nonrevision group $(p=0.05)$.

There were no differences between the two groups in terms of ASIA motor score at time of initial presentation, the use of allograft at the time of surgery, the use of chemotherapy or radiation therapy, and the use of preoperative embolization at the time surgery. The median number of vertebral levels involved in both groups was 1.0.

3.2. Table 2: Reasons for Revision. The primary reason for revision surgery was tumor progression. Approximately $67 \%$, $n=8$, of those needing a revision had tumor progression necessitating a need for a repeat surgery. The remaining $33 \%, n=4$, of revisions were for other reasons, including nonunion, failure of construct, and/or wound dehiscence.

3.3. Figure 1: Survival Curve (Revision versus No Revision). Figure 1 shows the estimated survival distributions for the two groups (revision versus no revision). The median overall survival for the group undergoing revision surgery was 3.0 years, while the median overall survival for the group that did not undergo a revision surgery was 1.5 years. Comparison of the survival curves between the two groups approached statistical significance $(p=0.105)$.

\subsection{Table 3: Univariate Regression Analysis for Undergoing} Revision. Of the individual prognostic factors evaluated, smoking status and use of adjuvant chemotherapy individually approached statistical significant association with probability of needing a revision. Smoking was found to be individually associated with the need for a revision, as 
TABLE 1: Patient and tumor characteristics.

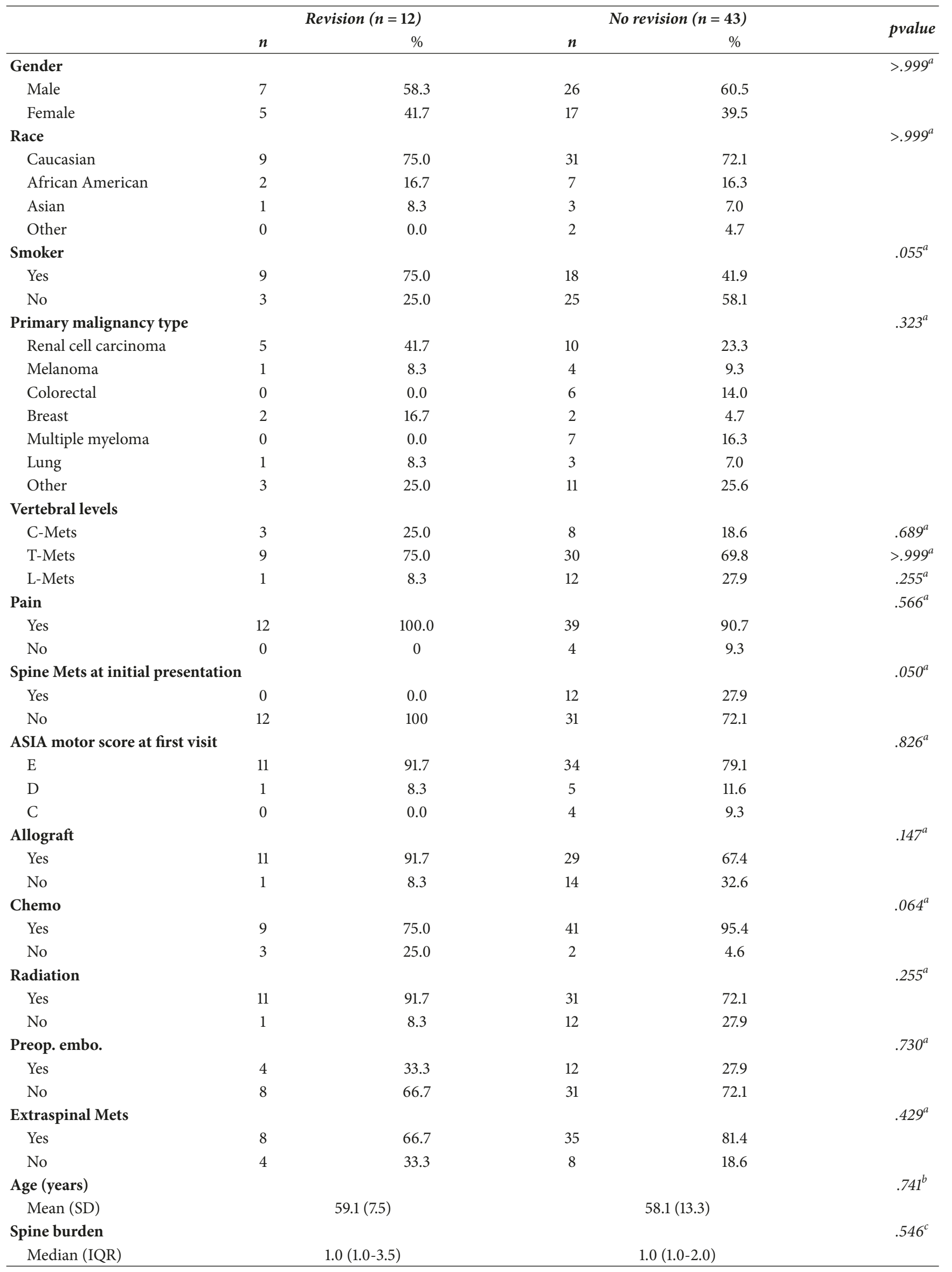


TABLE 2: Reasons for revision.

\begin{tabular}{lcc}
\hline & \multicolumn{2}{c}{ Revision } \\
Revision reason & $\boldsymbol{n}$ & $\%$ \\
\hline Tumor progression & 8 & 66.7 \\
Nonunion & 2 & 16.66 \\
Wound dehiscence & 1 & 8.33 \\
Construct failure & 1 & 8.33 \\
\hline
\end{tabular}

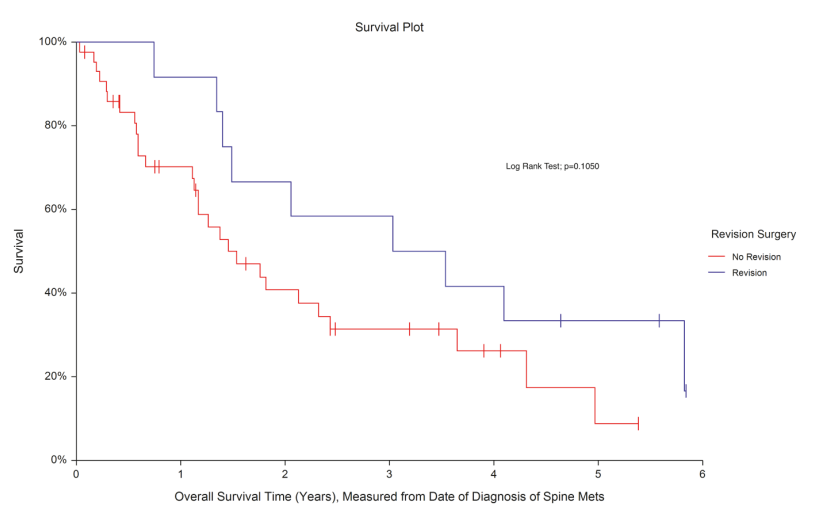

FIGURE 1

smoking increased the odds of needing a revision by 4.17 times compared to a nonsmoker $(p=0.052)$. Additionally, the odds of needing a revision in those who received adjuvant chemotherapy were 0.15 times the odds of needing a revision in those who received no adjuvant chemotherapy $(p=0.051)$.

3.5. Table 4: Multivariate Regression Analysis for Undergoing Revision. When smoking status and the use of chemotherapy were modeled together in a multivariate logistic regression model, chemotherapy drops from the model and use of smoking status becomes slightly attenuated (OR: 3.52, $p=$ 0.096).

In reviewing the ambulatory status of these patients, it was found that all twelve of the patients in our cohort were ambulatory either independently or with assistance prior to their operative intervention and immediately postoperatively. However, at their last clinic visit, it was found that only 5 of the 12 or $41.7 \%$ maintained their ability to ambulate.

\section{Discussion}

Surgical treatment of metastatic spine disease has been shown to improve pain, obtain control of disease, and improve quality of life [10-12]. Indications for surgical intervention for these patients continue to expand [7]. Additionally, superior adjuvant treatment options of treating metastatic disease have resulted in improved overall long-term survival of these patients [13].

Despite improvements in surgical control of spinal metastasis including obtaining circumferential spinal cord decompression and improved stabilization techniques [14], a certain proportion of patients undergoing surgical intervention of their metastatic spine will still need a revision surgery.
Revision surgery has been undertaken for surgical site infections and wound dehiscence, failure of instrumentation, and local recurrence/tumor progression [9]. In our study, 67\% of the patient population undergoing revision surgery had tumor progression. The majority of patients undergoing a revision had renal cell carcinoma as the dominant primary malignancy type $(41.7 \%)$, a cancer with an intermediate prognosis, which is also relatively radioresistant $[15,16]$.

Survival analysis revealed median survival in the revision group at 3.0 years, while the nonrevision group had 1.5 years. This approached statistical significance $(p=0.105)$. Metastasis to the spine carries a poor prognosis with time to death after surgery in most case series ranging from 11.3 to 15.4 months $[17,18]$. Patients with better natural history of their primary pathology can be expected to have a longer survival time. This allows for an inherent bias, where there is increased leadtime for tumor progression or instrumentation failure prior to the patient's death. However, if the natural prognosis is not favorable, despite tumor progression or hardware failure, these patients might not undergo revision surgery secondary to their overall poor prognosis, poor performance status (ECOG), or generally poor medical condition.

In exploring prognostic factors, smoking was found to have a notable effect on the odds of needing a revision surgery. After controlling for confounding variables, including the use of chemotherapy, smoking increased the odds of needing a revision by 3.52 times compared to a nonsmoker. Smoking has been found to have a negative impact in many disease pathologies: both oncological $[19,20]$ and nononcological entities, including decreasing rate of union [21]. The latter is especially important in the case of spinal fusions, which are frequently used as a treatment modality for stabilizing the affected spinal segment. Smoking can delay union and allow for the development of hardware failure, necessitating a revision. Additionally, smoking has also been implicated in wound healing complications that can also necessitate a revision [22].

Thus, patients undergoing primary surgical treatment for their spinal metastatic disease should also be counseled on the inherent dangers of smoking. Not only does smoking result in the production of carcinogens that can further increase the aggressiveness and spread of the primary tumor $[23,24]$ but also the odds of the patient undergoing a revision for their spinal metastasis also substantially increase.

The finding of a lower incidence of failure and decreased need for revision in patients receiving chemotherapy on univariate analysis is similarly relatable to the concept of natural history. Patients with systemic options for therapy likely have a more significant disease burden and may have a shorter life expectancy; this could potentially create a leadtime bias in which the group not receiving chemotherapy could have a higher likelihood to undergo a revision.

The natural history of patients with metastatic spinal tumors appears to be grim with survival only for a few years after diagnosis at best. Patients undergoing revision surgery tend to have comparable survival times to those not undergoing a revision. Thus, if a patient demonstrates indications for undergoing a revision surgery, that is, develops a hardware failure, and if their overall medical condition allows them 
TABLE 3: Univariate regression analysis for undergoing revision surgery.

\begin{tabular}{|c|c|c|c|c|}
\hline \multirow{2}{*}{$\begin{array}{l}\text { Variable } \\
\text { Age }\end{array}$} & \multirow{2}{*}{$\begin{array}{c}\text { Odds ratio } \\
1.007\end{array}$} & \multirow{2}{*}{$\frac{p \text { value }}{.803}$} & \multicolumn{2}{|c|}{ 95\% confidence interval } \\
\hline & & & 0.954 & 1.063 \\
\hline Race & & $>.999$ & & \\
\hline Caucasian versus other & $>999.999$ & .985 & $<0.001$ & $>999.999$ \\
\hline African American versus other & $>999.999$ & .985 & $<0.001$ & $>999.999$ \\
\hline Asian versus other & $>999.999$ & .984 & $<0.001$ & $>999.999$ \\
\hline \multicolumn{5}{|l|}{ Sex } \\
\hline Male versus female & 0.915 & .894 & 0.249 & 3.360 \\
\hline \multicolumn{5}{|l|}{ Smoker } \\
\hline Smoker versus nonsmoker & 4.166 & .052 & 0.987 & 17.589 \\
\hline Primary malignancy type & & .956 & & \\
\hline RCC versus other & 1.833 & .904 & 0.346 & 9.719 \\
\hline Melanoma versus other & 0.917 & .924 & 0.073 & 11.577 \\
\hline Colorectal versus other & $<0.001$ & .949 & $<0.001$ & $>999.999$ \\
\hline Breast versus other & 3.667 & .884 & 0.354 & 38.029 \\
\hline Multiple myeloma versus other & $<0.001$ & .946 & $<0.001$ & $>999.999$ \\
\hline Lung versus other & 1.222 & .916 & 0.091 & 16.429 \\
\hline \multicolumn{5}{|l|}{ Preop. ambulatory status } \\
\hline IND/AMB assist versus wheelchair/bed rest & $>999.999$ & .957 & $<0.001$ & $>999.999$ \\
\hline \multicolumn{5}{|l|}{ Radiation } \\
\hline Yes versus no & 4.258 & .187 & 0.495 & 36.659 \\
\hline \multicolumn{5}{|l|}{ Chemo } \\
\hline Yes versus no & 0.146 & .051 & 0.021 & 1.008 \\
\hline \multicolumn{5}{|l|}{ Extraspinal Mets } \\
\hline Presence versus absence & 0.457 & .282 & 0.110 & 1.901 \\
\hline
\end{tabular}

TABLE 4: Multivariable regression analysis for undergoing revision surgery.

\begin{tabular}{|c|c|c|c|c|}
\hline Variable & Odds ratio & $p$ value & \multicolumn{2}{|c|}{$\mathbf{9 5} \%$ confidence interval } \\
\hline \multicolumn{5}{|l|}{ Smoker } \\
\hline Smoker versus nonsmoker & 3.518 & .096 & 0.801 & 15.457 \\
\hline \multicolumn{5}{|l|}{ Adjuvant chemotherapy } \\
\hline Yes versus no & 0.196 & .110 & 0.027 & 1.450 \\
\hline
\end{tabular}

to undergo surgical intervention, they should be presented the option of undergoing a revision. Additionally, reoperation for recurrent metastatic tumors in patients with high-grade epidural spinal compression does not necessarily result in poor functional outcomes [25]. While smoking increases the odds of patients undergoing a revision procedure, a revision procedure does not necessarily decrease survival as compared to patients who only undergo a primary procedure.

For certain analyses, statistical significance was set at a $p$ value of 0.10 , due to the smaller sample size in certain categories. Additionally, due to the low prevalence of patients with this disease burden and the even lower prevalence of patients undergoing revision surgery, the patient numbers are, as expected, low and the study will be underpowered. Nonetheless, the relationships and conclusions postulated remain highly plausible. Additional studies with collaborations from multiple centers across vast geographical areas are needed to further confirm these findings. The biggest uncontrolled variable in studies such as this is the inconsistency of surgical indications and this may limit direct comparison of large groups of patients from multiple surgeons.

This study demonstrates some important differences when contrasted with a similar study by Quraishi et al. [14]. While the overall revision rate in this study was 2-fold higher, the mean survival time in the nonrevision group was two times longer and in the revision group it was nearly 4 times longer. This is unlikely to be a treatment effect but rather an observation that may be related to tumor-specific factors, surgical selection, or other indeterminate factors. Another major difference in this group is that 20 of 31 operations in the study by Quraishi [14] were performed in the same hospitalization (early reoperation) versus our study, where there were no early reoperations (mean of 685 days with a range of 102-1666 days). 


\section{Conclusion}

This study primarily compares the survival outcomes of patients undergoing revision surgery versus those undergoing only a primary procedure and explores characteristics that might influence patients needing a revision at a single institution. This particular study design may allow for more homogeneity of indications and technique. This will provide additional predictive information for the surgeon to counsel the patients and pursue revision surgery when indicated.

\section{Ethical Approval}

The study was approved by the institutional IRB.

\section{Conflicts of Interest}

The authors declare that they have no conflicts of interest.

\section{References}

[1] D. Choi, A. Crockard, C. Bunger et al., "Review of metastatic spine tumour classification and indications for surgery: The consensus statement of the Global Spine Tumour Study Group," European Spine Journal, vol. 19, no. 2, pp. 215-222, 2010.

[2] Z. L. Gokaslan, J. E. York, G. L. Walsh et al., "Transthoracic vertebrectomy for metastatic spinal tumors," Journal of Neurosurgery, vol. 89, no. 4, pp. 599-609, 1998.

[3] Y. Tokuhashi, H. Matsuzaki, S. Toriyama, H. Kawano, and S. Ohsaka, "Scoring system for the preoperative evaluation of metastatic spine tumor prognosis," The Spine Journal, vol. 15, no. 11, pp. 1110-1113, 1990.

[4] M. Wang, C. E. Bünger, H. Li et al., "Predictive value of tokuhashi scoring systems in spinal metastases, focusing on various primary tumor groups: Evaluation of 448 patients in the aarhus spinal metastases database," The Spine Journal, vol. 37, no. 7, pp. 573-582, 2012.

[5] K. Tomita, N. Kawahara, T. Kobayashi, A. Yoshida, H. Murakami, and T. Akamaru, "Surgical strategy for spinal metastases," The Spine Journal, vol. 26, no. 3, pp. 298-306, 2001.

[6] N. A. Quraishi and C. Esler, "Metastatic spinal cord compression," The BMJ: leading general medical journal, vol. 342, no. 7805, 2011.

[7] A. Amelot, L. Balabaud, D. Choi et al., "Surgery for metastatic spine tumors in the elderly. Advanced age is not a contraindication to surgery!," The Spine Journal, vol. 17, no. 6, pp. 759-767, 2015.

[8] C. Eap, E. Tardieux, O. Goasgen et al., “Tokuhashi score and other prognostic factors in 260 patients with surgery for vertebral metastases," Orthopaedics \& Traumatology: Surgery \& Research, vol. 101, no. 4, pp. 483-488, 2015.

[9] P. Padalkar and B. Tow, "Predictors of survival in surgically treated patients of spinal metastasis," Indian Journal of Orthopaedics, vol. 45, no. 4, pp. 307-313, 2011.

[10] Y. Tokuhashi, H. Kawano, S. Ohsaka et al., "[A scoring system for preoperative evaluation of the prognosis of metastatic spine tumor (a preliminary report)," Nihon Seikeigeka Gakkai Zasshi, vol. 63, pp. 482-489, 1989.

[11] E. A. Enkaoua, L. Doursounian, G. Chatellier, F. Mabesoone, T. Aimard, and G. Saillant, "Vertebral metastases: A critical appreciation of the preoperative prognostic Tokuhashi score in a series of 71 cases," The Spine Journal, vol. 22, no. 19, pp. 22932298, 1997.

[12] R. A. Patchell, P. A. Tibbs, W. F. Regine et al., "Direct decompressive surgical resection in the treatment of spinal cord compression caused by metastatic cancer: a randomised trial," The Lancet, vol. 366, no. 9486, pp. 643-648, 2005.

[13] H. Akram and J. Allibone, "Spinal surgery for palliation in malignant spinal cord compression," Clinical Oncology, vol. 22, no. 9, pp. 792-800, 2010.

[14] N. A. Quraishi, A. Rajabian, A. Spencer et al., "Reoperation rates in the surgical treatment of spinal metastases," The Spine Journal, vol. 15, no. 3, pp. 37-43, 2015.

[15] E. Maranzano, R. Bellavita, R. Rossi et al., "Short-course versus split-course radiotherapy in metastatic spinal cord compression: results of a phase III, randomized, multicenter trial," Journal of Clinical Oncology, vol. 23, no. 15, pp. 3358-3365, 2005.

[16] Y. Tokuhashi, H. Matsuzaki, H. Oda, M. Oshima, and J. Ryu, "A revised scoring system for preoperative evaluation of metastatic spine tumor prognosis," The Spine Journal, vol. 30, no. 19, pp. 2186-2191, 2005.

[17] C. E. Tatsui, D. Suki, G. Rao et al., "Factors affecting survival in 267 consecutive patients undergoing surgery for spinal metastasis from renal cell carcinoma," Journal of Neurosurgery: Spine, vol. 20, no. 1, pp. 108-116, 2014.

[18] J. N. Sellin, D. Suki, V. Harsh et al., "Factors affecting survival in 43 consecutive patients after surgery for spinal metastases from thyroid carcinoma," Journal of Neurosurgery: Spine, vol. 23, no. 4, pp. 419-428, 2015.

[19] T. M. Gibson, W. Liu, G. T. Armstrong et al., "Longitudinal smoking patterns in survivors of childhood cancer: An update from the Childhood Cancer Survivor Study," Cancer, vol. 121, no. 22, pp. 4035-4043, 2015.

[20] D. H. Howard, T. B. Richards, P. B. Bach, M. C. Kegler, and C. J. Berg, "Comorbidities, smoking status, and life expectancy among individuals eligible for lung cancer screening," Cancer, vol. 121, no. 24, pp. 4341-4347, 2015.

[21] R. Watkins, R. Watkins, and R. Hanna, "Non-union rate with stand-alone lateral lumbar interbody fusion," Medicine (United States), vol. 93, no. 29, p. e275, 2014.

[22] S. D. Glassman, S. C. Anagnost, A. Parker, D. Burke, J. R. Johnson, and J. R. Dimar, "The effect of cigarette smoking and smoking cessation on spinal fusion," The Spine Journal, vol. 25, no. 20, pp. 2608-2615, 2000.

[23] J. Liang, J. Shen, S. Chua et al., "Does intraoperative cell salvage system effectively decrease the need for allogeneic transfusions in scoliotic patients undergoing posterior spinal fusion? A prospective randomized study," European spine journal : official publication of the European Spine Society, the European Spinal Deformity Society, and the European Section of the Cervical Spine Research Society, vol. 24, no. 2, pp. 270-275, 2015.

[24] Z. Fu, M. J. Shrubsole, G. Li et al., "Interaction of cigarette smoking and carcinogen-metabolizing polymorphisms in the risk of colorectal polyps," Carcinogenesis, vol. 34, no. 4, pp. 779-786, 2013.

[25] I. Laufer, A. Hanover, E. Lis, Y. Yamada, and M. Bilsky, "Repeat decompression surgery for recurrent spinal metastases: Presented at the 2009 Joint Spine Section Meeting: Clinical article," Journal of Neurosurgery: Spine, vol. 13, no. 1, pp. 109-115, 2010. 


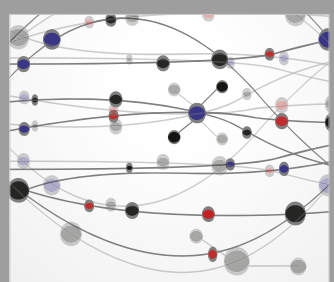

The Scientific World Journal
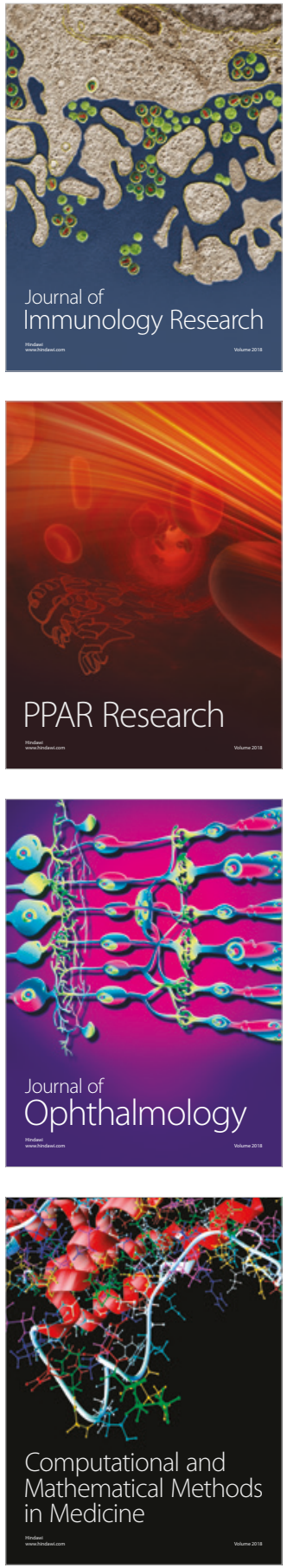

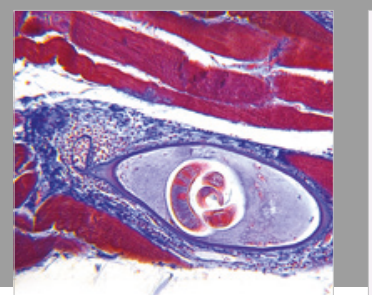

Gastroenterology Research and Practice

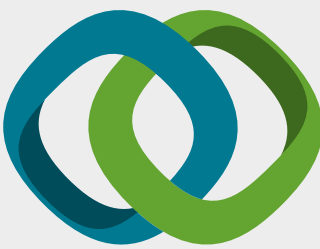

\section{Hindawi}

Submit your manuscripts at

www.hindawi.com
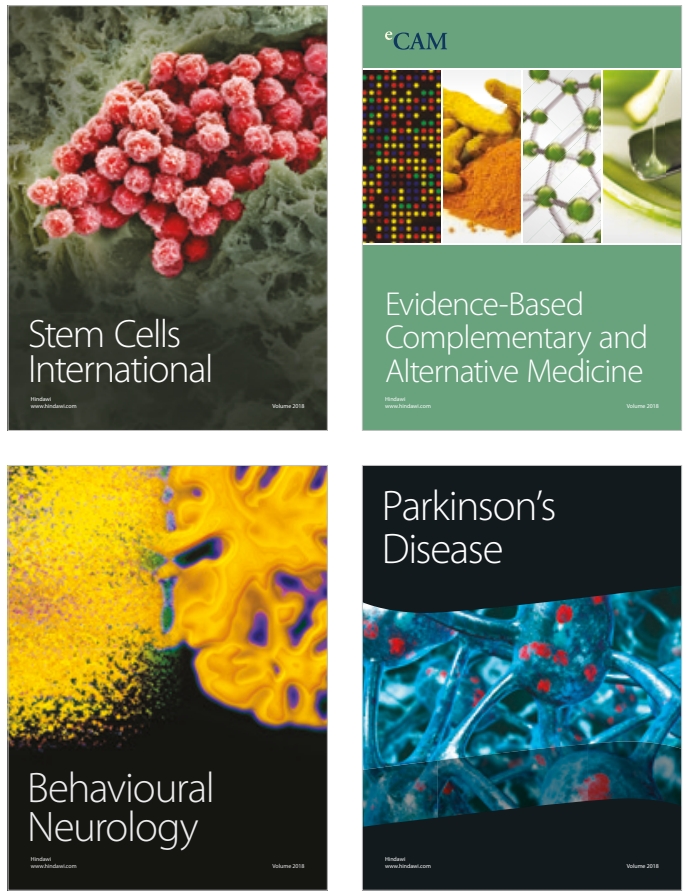

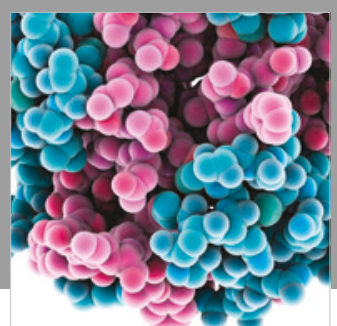

ournal of

Diabetes Research

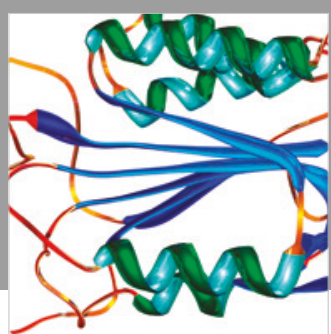

Disease Markers
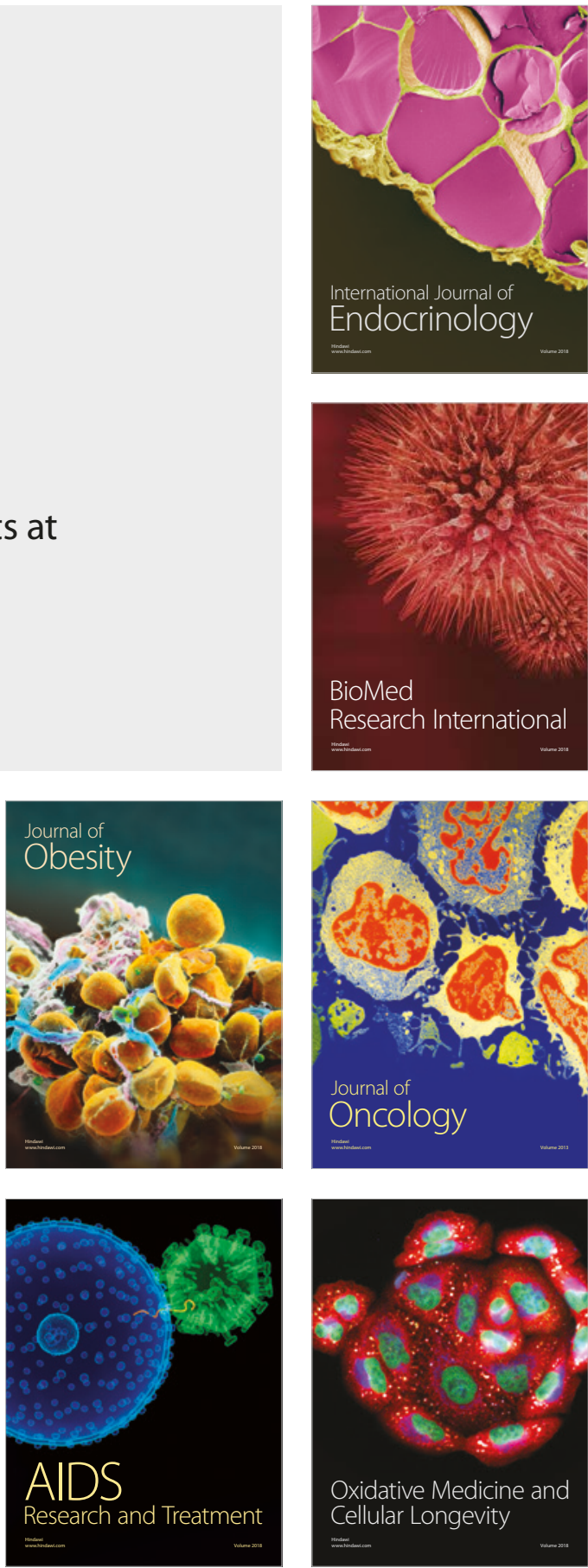